\title{
Fatores para não realização do parto via vaginal: revisão sistemática
}

\author{
Factors for not performing a vaginal delivery: systematic review \\ Factores para no realizar un parto vaginal: revisión sistemática
}

Recebido: 10/02/2022 | Revisado: 18/02/2022 | Aceito: 23/02/2022 | Publicado: 04/03/2022

Karla Salviani Azeredo dos Santos
ORCID: https://orcid.org/0000-0003-0291-1337
Universidade do Estado de Mato Grosso, Brasil
E-mail: karla.salviani @ unemat.br
Stefanny Maria Santana de Campos
ORCID: https://orcid.org/0000-0001-8667-3190
Universidade do Estado de Mato Grosso, Brasil
E-mail: stefanny.maria@ unemat.br
Danyella Rodrigues de Almeida
ORCID: https://orcid.org/0000 0003-1181-9321
Universidade do Estado de Mato Grosso, Brasil
E-mail: danyella.rodrigues@ unemat.br
Mayara de Oliveira Xaves
ORCID: https://orcid.org/0000-0001-9144-0244
Universidade do Estado de Mato Grosso, Brasil
E-mail: mayara.xaves@ unemat.br
Shaiana Vilella Hartwig
ORCID: https://orcid.org/0000-0003-4245-2163
Universidade do Estado de Mato Grosso, Brasil
E-mail: shaiana.hartwig@ unemat.br

\section{Resumo}

O Brasil vive uma epidemia de cirurgias cesáreas, superando estatisticamente o nível mundial. Neste sentido, a OMS declara que a taxa ideal para cesáreas seja de aproximadamente 10 a 15\%, sem condição justificável em qualquer região do mundo para que a taxa seja maior que esta. Nesta perspectiva, o Brasil situa-se com referida taxa de aproximadamente $56 \%$, sendo o serviço público $40 \%$, logo, é um dos países visado como percursor de partos cesáreos realizados de forma abusiva. Objetivo: Revisar os fatores que influenciam para a não realização do parto via vaginal, com base no comportamento materno e na assistência profissional, no Sistema Único de Saúde (SUS) do Brasil. Metodologia: Revisão sistemática de estudos publicados nas bases de dados LILACS, MEDLINE e BDENF através de descritores (parto normal, parto cesárea, comportamento materno, profissional da saúde e Brasil) catalogados no DeCS, em artigos publicados no período de 2016 a 2021. Resultados: Foram analisados 06 artigos. Com base no comportamento materno o fator medo é o principal contribuinte para a não realização do parto via vaginal; em contrapartida, quando relacionado a assistência profissional, ressalta-se o fator decisão. A maior parte dos estudos foram realizados na região nordeste, e as regiões Centro-oeste e Norte não dispuseram de pesquisas nesta linha de conteúdo. Considerações finais: Dentre os fatores encontrados, todos se comprometem à um contexto de realidade, podendo estar relacionado a experiências/assistências em tempos de pré-natal, parto e/ou puerpério, no contexto da vivência do parto via vaginal e/ou cirurgia cesárea.

Palavras-chave: Parto normal; Parto cesárea; Comportamento materno; Profissional da saúde; Brasil; Revisão.

\begin{abstract}
Brazil is experiencing an epidemic of cesarean surgery, statistically surpassing the world level. In this sense, WHO declares that the ideal rate for cesarean sections is approximately 10 to $15 \%$, with no justifiable condition in any region of the world for the rate to be higher than this. In this perspective, Brazil has the aforementioned rate of approximately $56 \%$, with the public service being $40 \%$, therefore, it is one of the countries targeted as a precursor of abusive cesarean deliveries. Objective: To review the factors that influence the non-accomplishment of vaginal delivery, based on maternal behavior and professional assistance, in the Brazilian Unified Health System (SUS). Methodology: Systematic review of studies published in LILACS, MEDLINE and BDENF databases using descriptors (normal delivery, cesarean delivery, maternal behavior, health professional and Brazil) cataloged in DeCS, in articles published from 2016 to 2021. Results: 06 articles were analyzed. Based on maternal behavior, the fear factor is the main contributor to the non-accomplishment of vaginal delivery; on the other hand, when related to professional assistance, the decision factor is highlighted. Most of the studies were carried out in the Northeast region, and the Midwest and North regions did not have research in this line of content. Final considerations: Among the factors found, all are committed to a context of reality, which may be related to experiences/assistance in prenatal, childbirth and/or
\end{abstract}


puerperium times, in the context of experiencing vaginal birth and/or cesarean surgery.

Keywords: Normal birth; Cesarean delivery; Maternal behavior; Health professional; Brazil; Review.

\section{Resumen}

Brasil vive una epidemia de cesáreas, superando estadísticamente el nivel mundial. En este sentido, la OMS declara que la tasa ideal de cesáreas es aproximadamente del 10 al 15\%, sin que exista condición justificable en ninguna región del mundo para que la tasa sea superior a esta. En esta perspectiva, Brasil tiene la tasa antes mencionada de aproximadamente el $56 \%$, siendo el servicio público el $40 \%$, por lo que es uno de los países apuntados como precursores de los partos por cesárea abusivos. Objetivo: Revisar los factores que influyen en la no realización del parto vaginal, basados en la conducta materna y la asistencia profesional, en el Sistema Único de Salud (SUS) de Brasil. Metodología: Revisión sistemática de estudios publicados en las bases de datos LILACS, MEDLINE y BDENF utilizando descriptores (parto normal, cesárea, conducta materna, profesional de la salud y Brasil) catalogados en DeCS, en artículos publicados de 2016 a 2021. Resultados: Se analizaron 06 artículos. Basado en el comportamiento materno, el factor miedo es el principal contribuyente a la no realización del parto vaginal; por otro lado, cuando se relaciona con la asistencia profesional, se destaca el factor de decisión. La mayoría de los estudios se realizaron en la región Nordeste, y las regiones Medio Oeste y Norte no contaron con investigaciones en esta línea de contenido. Consideraciones finales: Entre los factores encontrados, todos están comprometidos con un contexto de realidad, que puede estar relacionado con vivencias / asistencia en tiempos de prenatal, parto y / o puerperio, en el contexto de vivencia de parto vaginal y / o cesárea.

Palabras clave: Entrega normal; Parto por cesárea; Comportamiento materno; Profesional de la salud; Brasil; Revisión.

\section{Introdução}

Até os anos de 1.700, o parto era evento para ser resolvido entre mulheres envolvendo parteiras e mães, o qual se caracterizava unicamente em um processo natural (Brenes, 1991; Maldonado, 2002). O momento de atuação era da parturiente, as parteiras tinham um olhar cauteloso e mantinham a calma e dedicação, deixando acontecer por si só o parto (Maldonado, 2002). Iniciando as intervenções médicas com a inserção de cirurgias e materiais de apoio, como por exemplo, a utilização do fórceps (Maldonado, 2002), o cenário foi mudando, onde o personagem masculino se insere inaugurando o experimento clínico sob condição anatomopatológico, possibilitando a interrupção da gestação desde que algum sinal anatômico indicasse risco a vida da mulher (Brenes, 1991).

Com o avanço da tecnologia e surgimento de intervenções, a cesariana passa a ser destaque no mundo (Vendrúscolo et al.,2015), sendo que a ideia inicial do procedimento era a prevenção da morbimortalidade materna e perinatal (Betran et al., 2015). A prática tomou uma vasta proporção no Brasil. Neste sentido, a OMS (Organização Mundial da Saúde) declara sobre as taxas de cesáreas ressaltando que desde 1985 a comunidade médica internacional afirma que a taxa ideal para cesariana seja de aproximadamente 10 a 15\%, sem condição justificável em qualquer região do mundo para que a taxa seja maior (Organização Mundial da Saúde [OMS], 2015).

Sob reflexos de determinadas condutas, o Brasil ainda vive em uma epidemia de cirurgias cesáreas, (Rissin, \& Filho, 2018), situa-se com referida taxa de aproximadamente 56\%, havendo discrepante diferença entre serviços, sendo o público com faixa de $40 \%$ e o privado com $85 \%$ (Brasil, 2015). Tendo em vista que a estimativa de partos indiscriminados na América Latina é de 850.000 por ano, logo, o Brasil é um dos países visado como percursor de partos cesáreos realizados de forma abusiva (Anjos et al., 2014).

Com o intuito de mudar a realidade epidêmica, medidas estão sendo adotadas pelos órgãos competentes, como por exemplo, a elaboração e aprovação de diretrizes para atenção à gestante relacionada à operação cesariana (Brasil, 2016). Logo, a sugestão da OMS (2015) é que a Classificação de Robson seja usada a nível mundial, enquanto ainda está a preparar um manual padronizado.

Há um déficit no processo, falta à criação de um sistema de detecção de fatores influentes para o aumento das cesáreas, logo, com a implantação deste, possibilitaria melhor visibilidade padronizada do quadro e auxiliaria no melhor 
ajustamento da taxa ideal para esta via de parto (OMS, 2015). Diante dessa realidade, estudos apontam diversas versões sobre a situação, como por exemplo, segundo Brasil (2017) o avanço da obstetrícia contribuiu muito com a melhoria dos indicadores de mortalidade materna, porém, na atualidade tende a executar muitas intervenções desnecessárias rotineiramente.

As altas taxas de cesarianas no Brasil não podem ser associadas com o desejo ou solicitação das mulheres (Velho et al., 2014) e, um agravante que vem gerando uma indústria de cesáreas é a situação financeira destinada ao profissional, onde o ganho é consideravelmente maior realizando partos cesáreos, em menos tempo de serviço, quando comparado ao parto vaginal (Pimentel et al., 2016).

Ao analisar os fatores justificáveis para a realização do parto cesáreo sob ciência da avaliação individual de cada caso, os principais elencados são: apresentação pélvica, gestação múltipla, nascimento pré-termo, feto abaixo da idade gestacional, placenta prévia, acretismo placentário, preditores da progressão do trabalho de parto, infecção pelo HIV (vírus da imunodeficiência humana) - hepatite B e C - herpes simples, obesidade, operação cesariana prévia e ligação tubária (Brasil, 2015; Hospital Universitário Maria Aparecida Pedrossian [HUMAP], 2018). Quando comparado os discursos e desejos de puérperas referente à justificativa da cesárea, com os registros nos prontuários das mesmas, há contradição de informação (Viana et al., 2018).

A justificativa da escolha desta temática de trabalho visa aprofundar reflexões sobre o cenário atual das realizações de partos. Ao identificar os fatores que levam à realização do parto cesáreo possibilitará um olhar crítico sobre, para uma futura avaliação sobre a relevância de esses episódios estarem acontecendo. Na mesma perspectiva, o intuito é de identificar as possíveis fragilidades na assistência pública, caso tenha, e assim, com a ciência destas, gerará possibilidade de mobilização de entidades e profissionais responsáveis para uma reavaliação e mudança de condutas. Objetiva-se revisar os fatores que influenciam para a não realização do parto via vaginal, com base no comportamento materno e na assistência profissional, no Sistema Único de Saúde (SUS) do Brasil.

\section{Metodologia}

\subsection{Desenho De Estudo}

Pesquisa de revisão bibliográfica sistemática, do tipo descritivo, seguindo as seguintes etapas de acordo com Mendes, Silveira e Galvão (2008): definição do objeto e formulação da questão norteadora; busca nas bases de dados e delimitação dos critérios e inclusão; categorização dos estudos; análise crítica dos estudos selecionados; interpretação dos resultados; e apresentação da síntese do conhecimento.

\subsection{Questão norteadora}

Quais os fatores que levam a não realização do parto via vaginal, com base no comportamento materno e na assistência profissional, no Sistema Único de Saúde (SUS) do Brasil?

\subsection{Bases de dados eletrônicas}

A pesquisa bibliográfica foi realizada no portal da BVS (Biblioteca Virtual em Saúde), incluindo as bases de dados eletrônicas:

(1) Literatura Latino-americana e do Caribe em Ciências da Saúde - LILACS;

(2) Medical Literature Analysis and Retrieved System Online - MEDLINE; e

(3) Base de Dados em Enfermagem - BDENF. 


\subsection{Estratégia de busca}

As buscas foram direcionadas através de descritores catalogados no DeCS (Descritor em Ciências da Saúde) - parto normal, parto cesárea, comportamento materno, profissional da saúde e Brasil (com o intuito de filtrar estudos realizados no Brasil atendendo ao objetivo proposto) -, no idioma português, contidos no título ou no resumo dos estudos. Como operador booleano, utilizou-se "AND".

A combinação de termos utilizados nas bases de dados foi feita de forma controlada e sistematizada. Em todos os cruzamentos foram filtrados: textos completos; idioma português, inglês e espanhol; e ano de publicação nos últimos cinco anos (2016-2021), o período escolhido é por não ter revisões nesse tempo A identificação e inclusão dos artigos ocorreu no primeiro semestre de 2021, nos meses de fevereiro, março, abril.

\subsection{Seleção e análise das publicações}

Construiu-se uma tabela para auxiliar na seleção dos artigos, o qual compõe as seguintes informações: autor; ano de publicação; base de dados; periódico de publicação; título; objetivo; desenho de estudo; método de coleta de dados; principais resultados e conclusão.

Os critérios de inclusão para os estudos foram artigos completos do tipo original que contém nos resultados algum assunto que aborda sobre motivos que influenciam a não realização do parto via vaginal, com base no comportamento materno e/ ou na assistência profissional, no Sistema Único de Saúde (SUS) no Brasil; publicados entre abril de 2016 a abril de 2021, nos idiomas português, inglês e/ou espanhol; e indexados em uma das bases de dados anteriormente citadas. Foram excluídos artigos duplicados, resumos, editoriais, artigos de revisão de literatura (integrativa ou sistemática), síntese de experiências, relato de experiência, estudos de caso, cartas ao editor, anais de eventos científicos, tese, dissertação, e artigos que não contemplam o objeto de estudo proposto.

Após a busca encontrou-se 68 (sessenta e oito) bibliografias, feita a primeira análise (leitura de títulos e resumos) identificou-se 08 (oito) artigos científicos demonstrando fatores para a não realização do parto via vaginal. Ao realizar a segunda análise, leitura completa dos estudos, 06 (seis) foram selecionados para a elaboração desta revisão de literatura científica, e 02 (dois) foram excluídos devido não atenderem ao objetivo proposto. Os resultados obtidos com a aplicação da estratégia de busca descrita estão apresentados no quadro lógico do estudo (Figura 1). Os estudos são apresentados em quadros segundo o tipo de estudo (quantitativo ou qualitativo), com as seguintes informações: cronologia de publicação, autores, título, objetivo, desenho de estudo, número de participantes, método de coleta de dados, principais resultados e conclusão.

Sob análise crítica e síntese dos manuscritos dos estudos, os resultados encontrados foram separados em duas categorias: "Comportamento Materno" e "Assistência Profissional", foi analisado os conteúdos para chegar as categorias. Posteriormente foram elencadas palavras-chaves elencadas pelos autores que retratam resumidamente fatores que influenciam a não realização do parto via vaginal. Para a organização desses resultados, construiu-se uma nuvem de palavras para cada categoria; foi utilizado o programa informático chamado Wordle®, possibilitando organizar as palavras em tamanhos de acordo com a quantidade de menções feitas, quanto maior o tamanho mais citações apresentou a palavra. Para estruturar a discussão, os resultados foram esquematizados em duas categorias: Fatores para a não realização do parto via vaginal com base no comportamento materno; Fatores para a não realização do parto via vaginal com base na assistência profissional.

\section{Resultados e Discussão}

As combinações com os descritores e operadores booleanos resultaram em doze combinações, das quais em duas não obteve-se resultado (escolha do parto AND comportamento materno AND Brasil e escolha da via de parto AND assistência profissional AND Brasil), e a combinação que demonstrou mais resultados foi um total de 22 artigos (parto normal AND 
assistência profissional AND Brasil). A base de dados que mais se fez presente nos artigos foi a LILACS em 51 artigos, seguindo respectivamente BDENF em 30 artigos e MEDLINE em 11 artigos (Tabela 1).

Tabela 1 - Combinações de descritores e operadores booleanos para seleção de publicações científicas sobre motivos que influenciam a não realização do parto via vaginal, com base no comportamento materno e/ ou na assistência profissional, no Sistema Único de Saúde (SUS) no Brasil, publicações de 2016 a 2021.

\begin{tabular}{|c|c|c|c|c|}
\hline \multirow[t]{2}{*}{ Descritores e operadores booleanos } & \multicolumn{3}{|c|}{$\begin{array}{l}\text { Bases de dados pesquisada } \\
\end{array}$} & \multirow[t]{2}{*}{ Total de artigos } \\
\hline & LILACS & MEDLINE & BDENF & \\
\hline Parto normal AND assistência profissional AND Brasil & 17 & 02 & 14 & 22 artigos \\
\hline Parto normal AND comportamento materno AND Brasil & 02 & 01 & 01 & 03 artigos \\
\hline Parto cesárea AND assistência profissional AND Brasil & 12 & 03 & 07 & 16 artigos \\
\hline Parto cesárea AND comportamento materno AND Brasil & 01 & 00 & 01 & 01 artigo \\
\hline Escolha do parto AND comportamento materno AND Brasil & - & - & - & 00 artigo \\
\hline $\begin{array}{l}\text { Escolha da via de parto AND assistência profissional AND } \\
\text { Brasil }\end{array}$ & - & - & - & 00 artigo \\
\hline Escolha de parto AND profissional da saúde AND Brasil & 07 & 01 & 02 & 08 artigos \\
\hline $\begin{array}{l}\text { Fatores AND parto cesárea AND puérperas AND } \\
\text { profissional da saúde AND Brasil }\end{array}$ & 03 & 01 & 01 & 05 artigos \\
\hline Fatores AND escolha de parto AND puérperas AND Brasil & 01 & 00 & 01 & 01 artigo \\
\hline $\begin{array}{l}\text { Fatores AND escolha de parto AND assistência profissional } \\
\text { AND Brasil }\end{array}$ & 02 & 01 & 00 & 03 artigos \\
\hline $\begin{array}{l}\text { Fatores AND cesárea AND assistência profissional AND } \\
\text { Brasil }\end{array}$ & 05 & 02 & 02 & 8 artigos \\
\hline $\begin{array}{l}\text { Fatores AND cesárea AND comportamento materno AND } \\
\text { Brasil }\end{array}$ & 01 & 00 & 01 & 01 artigo \\
\hline
\end{tabular}

Fonte: Autores. 
Figura 1 - Quadro lógico da revisão sistemática de publicações científicas sobre motivos que influenciam a não realização do parto via vaginal, com base no comportamento materno e/ ou na assistência profissional, no Sistema Único de Saúde (SUS) no Brasil, publicações de 2016 a 2021.

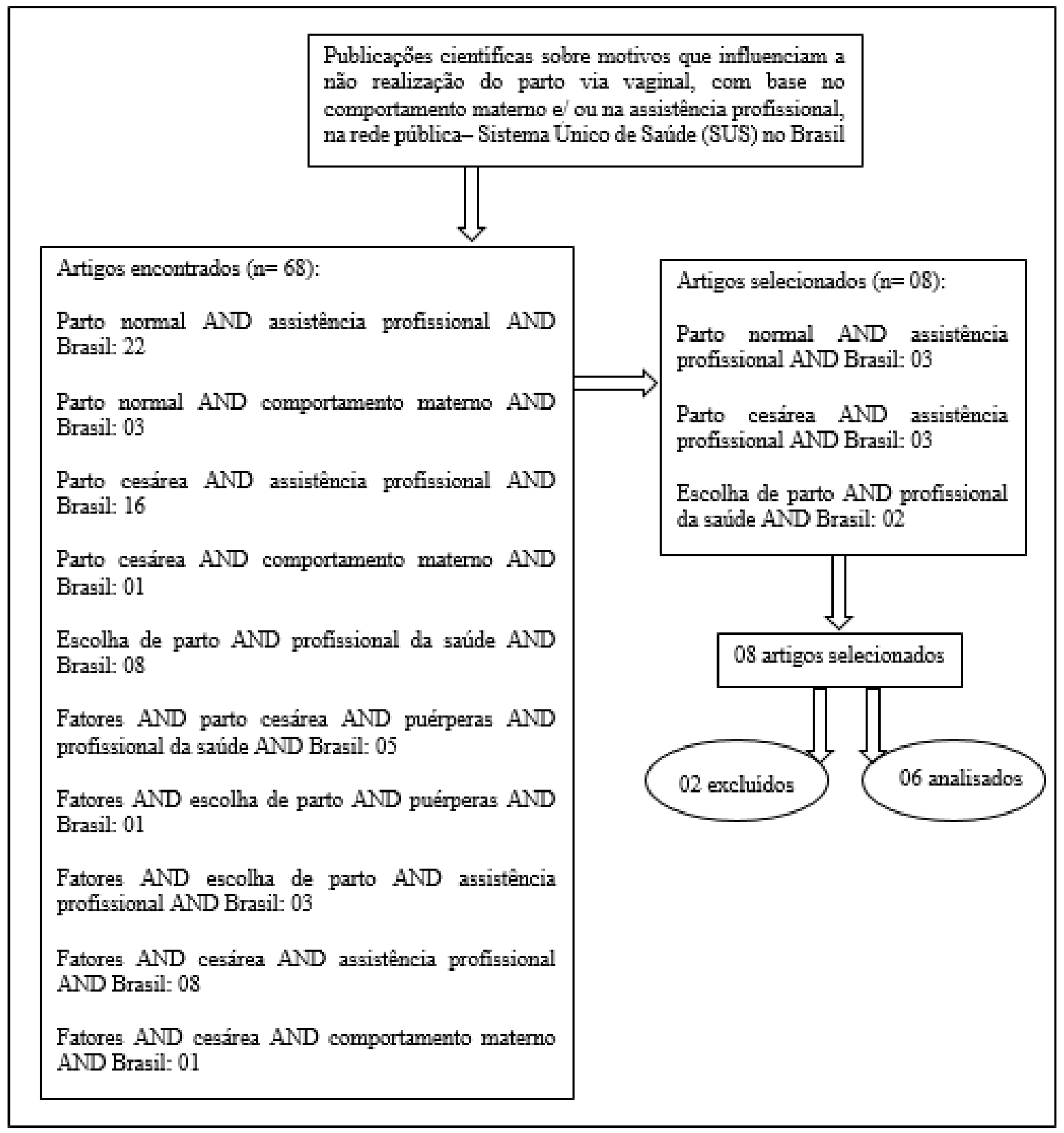

Fonte: Autores.

Foram encontrados quatro estudos de cunho qualitativo, sob o tipo de pesquisa descritivo exploratório e apenas um etno-metodológica. Para a coleta de dados, em três pesquisas foi utilizado um instrumento (entrevista, formulário, roteiro) semiestruturado, e em uma pesquisa utilizou-se entrevistas individuais através de relatos comunicativos (Quadro 1).

Duas pesquisas foram realizadas no ano de 2018 e as demais, uma em 2013 e a outra em 2015.Cada estudo apresentou quantidade de participantes diferentes, sendo, 12 enfermeiras, 13 puérperas (após o parto vaginal), 10 puérperas (após o parto cesárea) e 07 mulheres (em dois momentos, primeiros gestantes e depois puérperas). Os locais onde foram desenvolvidas as pesquisas foram as Unidade de Estratégia da Saúde da Família e em Maternidades (Quadro 1). 
Os estudos evidenciam fatores para a não realização do parto via vaginal em diversos aspectos, onde abordam condições relacionadas ao comportamento materno, do ponto de vista de puérperas após parto normal e cesárea, e também relacionado a assistência profissional (Quadro 1). Há semelhança entre dois estudos ao pontuarem a relação entre a gestante, a informação e o profissional , onde cada um aborda reflexos diferentes no que diz respeito a realização das vias de parto; em contrapartida, as demais pesquisas evidenciam diferenças ao mencionar que profissionais da atenção primária identificam sentimento de insegurança e medo nas gestantes, para o parto vaginal; parturientes com experiência do parto normal mencionam insatisfação relacionado à verticalização das relações, ausência de acompanhamento profissional e banalização da dor durante o parto; com relação à cesariana, puérperas demonstram que a decisão pela via de parto foi exclusivamente do profissional médico, logo, o cuidado da equipe e transmissão de segurança corrobora para o conformismo da gestante para o parto cesárea (Quadro 1). 


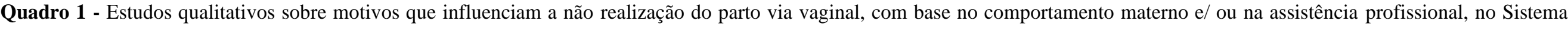
Único de Saúde (SUS) no Brasil, publicações de 2016 a 2021.

\begin{tabular}{|c|c|c|c|c|c|c|}
\hline $\begin{array}{l}N^{\circ} \text { do } \\
\text { artigo }\end{array}$ & $\begin{array}{l}\text { Autores } \\
\text { Ano de } \\
\text { Publicação }\end{array}$ & Título & Objetivo & $\begin{array}{l}\text { Desenho de estudo } \\
\text { Número de participantes } \\
\text { Método de coleta dos dados } \\
\end{array}$ & Principais resultados & Conclusão \\
\hline 1 & $\begin{array}{l}\text { Santos } \\
\text { Fabbro. } \\
2018\end{array}$ & $\begin{array}{l}\text { A difícil tarefa } \\
\text { de escolher o } \\
\text { parto natural }\end{array}$ & $\begin{array}{l}\text { Compreender } \\
\text { motivações de } \\
\text { mulheres usuárias } \\
\text { do Epi-no à escolha } \\
\text { do parto natural } \\
\text { entre 2013-2015, } \\
\text { descrevendo } \\
\text { elementos } \\
\text { facilitadores }(\mathrm{EF})\end{array}$ & $\begin{array}{l}\text { Estudo qualitativo } \\
\mathrm{N} \text { : } 07 \text { gestantes e posteriormente } \\
\text { puérperas. } \\
\text { Utilizou-se } \\
\text { o Feminismo Dialógico } \\
\text { a Metodologia Comunicativa /MC } \\
\text { como referenciais teórico- } \\
\text { metodológicos, por meio de } \\
\text { relatos de } 7 \text { mulheres, gravados } \\
\text { em áudio, transcritos e analisados. }\end{array}$ & $\begin{array}{l}\text { Idade 22-38 anos; todos partos } \\
\text { vaginais. Elaboraram-se } 14 \text { temáticas, } \\
\text { agrupadas em EF e ED (elementos } \\
\text { dificultadores) Conhecendo sua } \\
\text { história; apoio } \\
\text { família/profissional/doula; Preparação } \\
\text { física e psicológica para o parto; e, } \\
\text { reconhecendo as fases do trabalho de } \\
\text { parto. Essas foram reagrupados em } 2 \\
\text { categorias da MC mundo da vida e } \\
\text { sistema e apontaram recomendações } \\
\text { em consenso com as mulheres. }\end{array}$ & $\begin{array}{l}\text { As pessoas (mundo da vida) } \\
\text { possuem papel importanter como } \\
\text { transformadoras sociais. Motivadas e } \\
\text { informadas encontram encorajamento } \\
\text { e segurança, produzindo mudanças e } \\
\text { demonstrando que crenças, culturas, } \\
\text { medos em torno do parto podem ser } \\
\text { desmistificados. }\end{array}$ \\
\hline 2 & $\begin{array}{l}\text { Albuquerque, } \\
\text { Mendonça, } \\
\text { Guerra, Silva, e } \\
\text { Lins. } \\
2019\end{array}$ & $\begin{array}{l}\text { Representaçõe } \\
\mathrm{s} \text { sociais de } \\
\text { enfermeiras da } \\
\text { atenção básica } \\
\text { sobre o parto } \\
\text { normal }\end{array}$ & $\begin{array}{lr}\text { Identificar } & \text { as } \\
\text { representações } & \\
\text { sociais } & \text { de } \\
\text { enfermeiras } & \text { da } \\
\text { atenção } & \text { básica } \\
\text { sobre parto normal }\end{array}$ & $\begin{array}{l}\text { Estudo descritivo, exploratório, } \\
\text { qualitativo. } \\
\text { N: } 12 \text { enfermeiras. } \\
\text { Foram realizadas entrevistas } \\
\text { semi-estruturadas, guiadas pela } \\
\text { questão norteadora Fale-me sobre } \\
\text { parto normal }\end{array}$ & 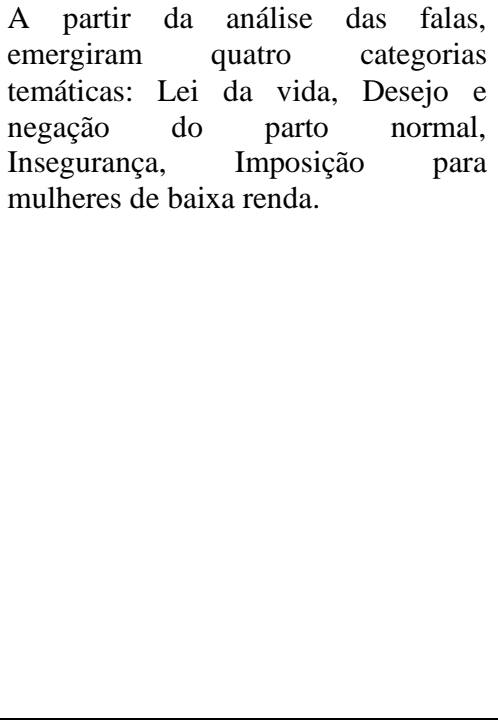 & $\begin{array}{l}\text { As representações que emergiram das } \\
\text { falas das enfermeiras apresentam o } \\
\text { parto normal como um evento natural, } \\
\text { permeado pelo auxílio divino, trazendo } \\
\text { o desejo em vivenciá-lo. Entretanto, } \\
\text { este fato não é suficiente para que haja } \\
\text { uma escolha por este tipo de parto, } \\
\text { devido a outros fatores, como a } \\
\text { insegurança presente em diferentes } \\
\text { momentos e aspectos, bem como a } \\
\text { compreensão de que o parto normal é } \\
\text { imposto nos serviços públicos de } \\
\text { saúde. Dessa forma, compreender as } \\
\text { representações sociais do parto normal, } \\
\text { pela perspectiva da enfermagem } \\
\text { atuante na atenção básica, propicia um } \\
\text { olhar sob a educação em saúde no pré- } \\
\text { natal, onde são reelaborados os } \\
\text { conhecimentos que envolvem este } \\
\text { evento (AU). }\end{array}$ \\
\hline 3 & $\begin{array}{l}\text { Barral et al. } \\
2020\end{array}$ & $\begin{array}{l}\text { Parto } \\
\text { cirúrgico: } \\
\text { múltiplas }\end{array}$ & $\begin{array}{l}\text { Conhecer as } \\
\text { experiências } \\
\text { de mulheres que }\end{array}$ & $\begin{array}{l}\text { Estudo qualitativo } \\
\mathrm{N}: 10 \text { puérperas. }\end{array}$ & $\begin{array}{l}\text { As experiências das mulheres sobre } \\
\text { o parto cirúrgico são permeadas } \\
\text { pelo medo, atrelado principalmente }\end{array}$ & 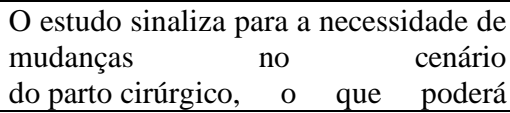 \\
\hline
\end{tabular}




\begin{tabular}{|c|c|c|c|c|c|c|}
\hline & & $\begin{array}{l}\text { experiências } \\
\text { de mulheres }\end{array}$ & $\begin{array}{l}\text { vivenciaram } \\
\text { o parto cirúrgico }\end{array}$ & $\begin{array}{l}\text { Foram realizadas entrevistas com } \\
\text { dez mulheres que } \\
\text { experienciaram parto cirúrgico } \\
\text { em uma maternidade de Salvador, } \\
\text { Bahia, Brasil. Posteriormente os } \\
\text { dados foram sistematizados } \\
\text { mediante a Análise Temática de } \\
\text { Bardin. }\end{array}$ & 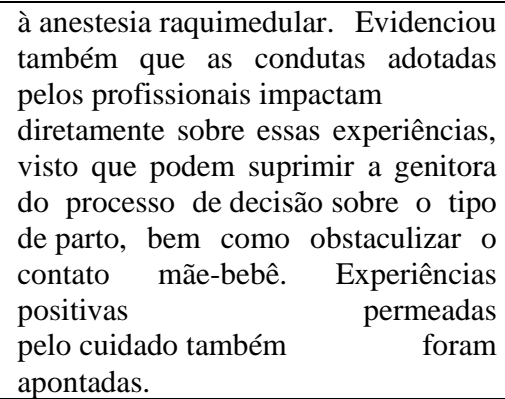 & $\begin{array}{l}\text { contribuir para uma prática } \\
\text { profissional que prioriza a qualidade da } \\
\text { assistência ofertada e favorece } \\
\text { o empoderamento das mulheres. }\end{array}$ \\
\hline 4 & $\begin{array}{l}\text { Bomfim et al. } \\
2021\end{array}$ & $\begin{array}{l}\text { Percepções de } \\
\text { mulheres } \\
\text { sobre a } \\
\text { assistência de } \\
\text { enfermagem } \\
\text { durante o } \\
\text { parto normal }\end{array}$ & $\begin{array}{l}\text { Conhecer a percepç } \\
\text { ão de mulheres sobr } \\
\text { e a assistência de } \\
\text { Enfermagem recebi } \\
\text { da durante o } \\
\text { processo de parto } \\
\text { normal }\end{array}$ & $\begin{array}{l}\text { Estudo descritivo com abordagem } \\
\text { qualitativa. } \\
\text { N: } 13 \text { puérperas. } \\
\text { Efetuada } \\
\text { duas maternidades públicas de } \\
\begin{array}{l}\text { Salvador, Bahia, Brasil. A coleta } \\
\text { de dados foi efetuada } \\
\text { por entrevista }\end{array}\end{array}$ & $\begin{array}{lr}\text { Emergiram duas } & \text { categorias } \\
\text { científicas assistência } & \text { de } \\
\text { Enfermagem permeada } & \text { por } \\
\text { satisfação; e assistência permeada por } \\
\text { relações verticais e sentimentos de } \\
\text { abandono }\end{array}$ & $\begin{array}{l}\text { A percepção das mulheres sobre } \\
\text { a assistência de Enfermagem recebida } \\
\text { durante o processo de parto normal foi } \\
\text { dicotômica. }\end{array}$ \\
\hline
\end{tabular}

Fonte: Autores.

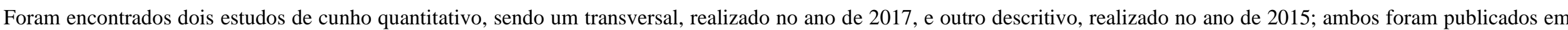

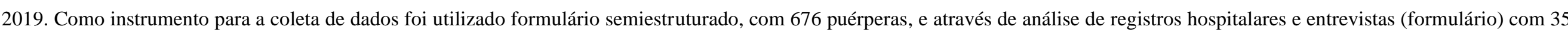
primíparas (Quadro 2).

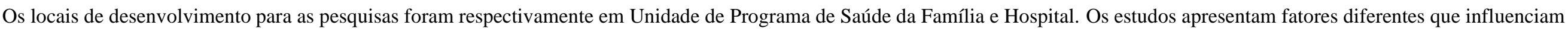

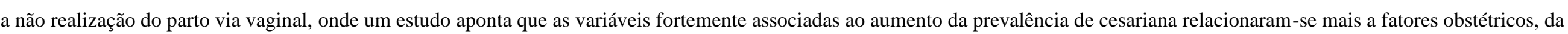

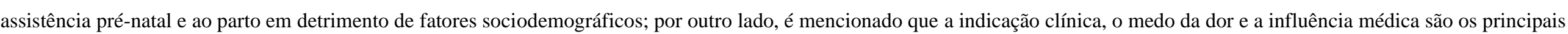
fatores que levam a parturiente a se submeter ao parto cesárea (Quadro 2). 


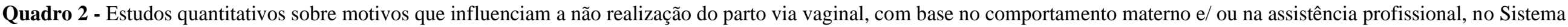
Único de Saúde (SUS) no Brasil, publicações de 2016 a 2021.

\begin{tabular}{|c|c|c|c|c|c|c|}
\hline $\begin{array}{l}\mathrm{N}^{0} \text { do } \\
\text { artigo }\end{array}$ & $\begin{array}{c}\text { Autores } \\
\text { Ano de } \\
\text { Publicação }\end{array}$ & Título & Objetivo & $\begin{array}{l}\text { Desenho de estudo } \\
\text { Número de participantes } \\
\text { Método de Coleta de dados }\end{array}$ & Principais resultados & Conclusão \\
\hline 1 & $\begin{array}{l}\text { Rasador } \\
\text { e Abegg. } \\
2019\end{array}$ & $\begin{array}{l}\text { Fatores } \\
\text { associados à } \\
\text { via de parto } \\
\text { em um } \\
\text { município da } \\
\text { região } \\
\text { nordeste do } \\
\text { Estado do Rio } \\
\text { Grande do } \\
\text { Sul, Brasil }\end{array}$ & $\begin{array}{l}\text { Investigar os fatores } \\
\text { associados à via } \\
\text { de nascimento em } \\
\text { um hospital de } \\
\text { atendimento misto, } \\
\text { público e privado, da } \\
\text { região nordeste do Rio } \\
\text { Grande do Sul. }\end{array}$ & $\begin{array}{l}\text { Estudo transversal } \\
\text { N: } 676 \text { puérperas. } \\
\begin{array}{lr}\text { Os dados foram obtidos } \\
\text { de registros hospitalares e } \\
\text { entrevistas com } \\
\text { as mulheres logo r após } \\
\text { o parto na maternidade }\end{array}\end{array}$ & 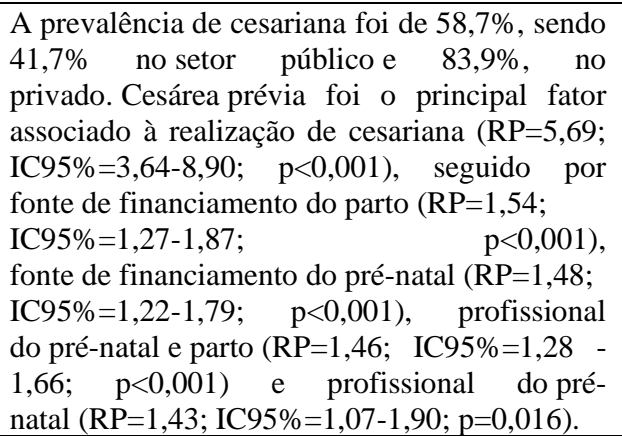 & $\begin{array}{l}\text { As elevadas taxas de cesariana } \\
\text { identificadas neste estudo foram } \\
\text { associadas principalmente à } \\
\text { realização de cesárea prévia. Os } \\
\text { achados indicam a necessidade } \\
\text { de mudança no modelo de assistência } \\
\text { ao parto no município, o qual é } \\
\text { altamente medicalizado, centrado no } \\
\text { profissional médico } \\
\text { na atenção hospitalar. }\end{array}$ \\
\hline 2 & $\begin{array}{l}\text { Silva, Silva e } \\
\text { Melo. } \\
2019\end{array}$ & $\begin{array}{ll}\text { Autonomia } & \text { da } \\
\text { gestante na } \\
\text { escolha do } \\
\text { tipo de parto }\end{array}$ & $\begin{array}{l}\text { Identificar a } \\
\text { preferência da gestante } \\
\text { primigesta quanto à via } \\
\text { de parto, conhecer os } \\
\text { fatores que influenciam } \\
\text { a sua tomada de } \\
\text { decisão nessa escolha, } \\
\text { e as } \\
\text { suas expectativas em } \\
\text { relação } \\
\text { ao parto mediante a via } \\
\text { escolhida. }\end{array}$ & $\begin{array}{l}\text { Estudo descritivo com } \\
\text { abordagem quantitativa } \\
\mathrm{N}: 35 \text { primigestas. } \\
\text { Formulário semiestruturado }\end{array}$ & $\begin{array}{l}\text { Predominou a escolha pela via } \\
\text { de parto vaginal, } \\
\text { de expectativas positivas em relação a via } \\
\text { escolhida e a não influência de terceiros sobre } \\
\text { a tomada de decisão. }\end{array}$ & $\begin{array}{l}\text { A decisão da mulher na escolha da } \\
\text { via de parto está se transformando } \\
\text { em uma preferência e não apenas em } \\
\text { uma indicação profissional, o que } \\
\text { ressalta a importância da educação } \\
\text { em saúde na consulta pré-natal. }\end{array}$ \\
\hline
\end{tabular}

Fonte: Autores. 
Dentre os seis artigos analisados, obtemos três estudos realizados na região Nordeste, um realizado na região Sul e dois realizados na região Sudeste; a região Centro-oeste e Norte não dispôs de pesquisas realizadas nesta linha de conteúdo (Figura 2).

Figura 2 - Regiões em que foram realizadas as pesquisas sobre motivos que influenciam a não realização do parto via vaginal, com base no comportamento materno e/ ou na assistência profissional, no Sistema Único de Saúde (SUS) no Brasil, publicações de 2016 a 2021.

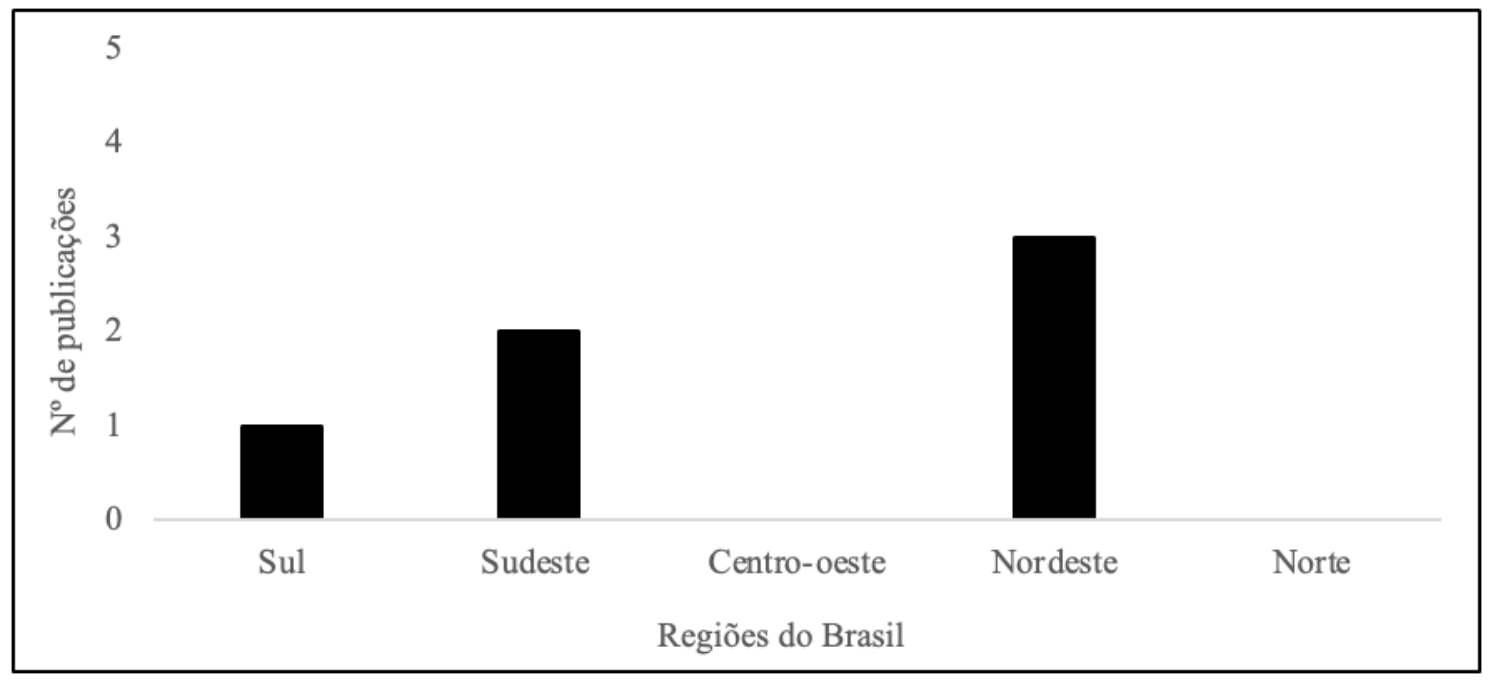

Fonte: Autores.

A categoria "Comportamento Materno" evidência fatores que influenciam a não realização do parto via vaginal com base no que diz respeito a mulher que se submete ao processo de parturição; dentre os fatores retratados na nuvem de palavras, totalizaram 24 palavras, sendo insegurança (Albuquerque, Mendonça, Guerra, Silva, \& Lins, 2019), medo (Albuquerque et al., 2019; Silva, Silva et al., 2019), desconforto (Bomfim et al, 2021), abandono (Bomfim et al, 2021), negligência (Bomfim et al, 2021), insatisfação (Bomfim et al, 2021), confiança (Barral et al., 2020), conformismo (Barral et al., 2020), concordância (Barral et al., 2020), conhecimento (Barral et al., 2020), desinformação (Barral et al., 2020), desejo (Rasador et al., 2019), obstétrico (Rasador et al., 2019), sociodemográficos (Rasador et al., 2019), escolaridade (Rasador et al., 2019), cesárea prévia (Rasador et al., 2019), ruptura uterina (Rasador et al., 2019), pseudoautonomia (Silva et al., 2019), idealização (Santos \& Fabbro, 2018), imaginário (Santos et al.,, 2018), vulnerabilidades (Santos et al., 2018), fragilidades (Santos et al., 2018), cultura (Santos et al., 2018) e informação (Santos et al., 2018) (Figura 3). 
Figura 3 - Palavras-chave que retratam resumidamente fatores que influenciam a não realização do parto via vaginal com base no comportamento materno, no Sistema Único de Saúde (SUS) no Brasil; publicações de 2016 a 2021.

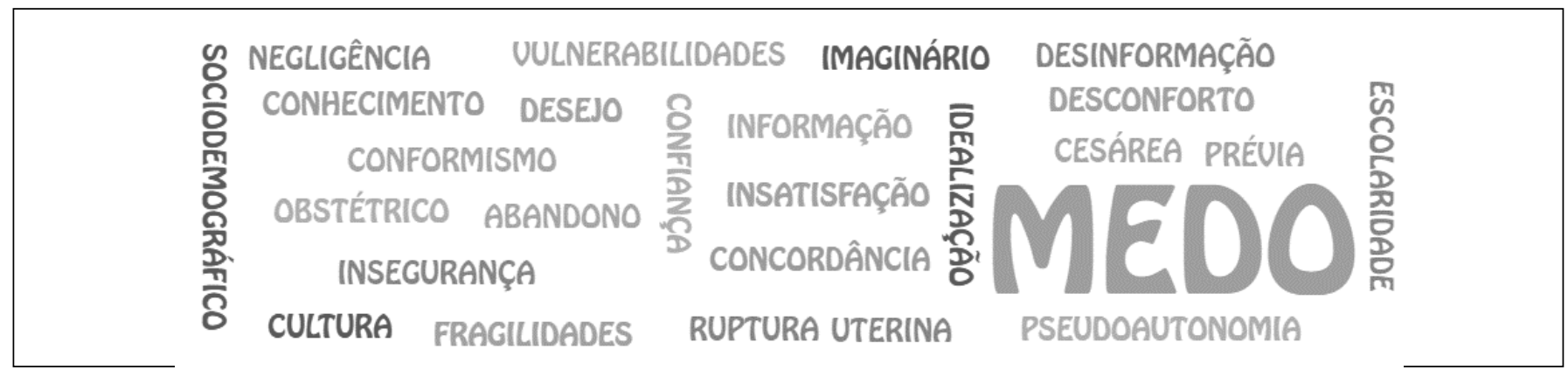

Fonte: Autores.

Os fatores que influenciam para a não realização do parto via vaginal relacionados a categoria "Assistência Profissional" - a qual refere às situações envoltas às competências dos profissionais da saúde que prestam assistência à gestante, resultaram em 18 menções sendo imposição (Albuquerque et al., 2019), decisão (Barral et al., 2020; Silva et al., 2019), avaliação clínica (Barral et al., 2020), cuidado (Barral et al., 2020), preocupação (Barral et al., 2020), carinho (Barral et al., 2020), atenção (Barral et al., 2020), assistência humanizada (Barral et al., 2020), tranquilizar (Barral et al., 2020), expertise (Barral et al., 2020), pré-natal (Rasador \& Abegg, 2019), informação (Silva et al., 2019), influência (Silva et al., 2019), indicação clínica (Silva et al., 2019), recomendações (Silva et al., 2019), violência obstétrica (Santos \& Fabbro, 2018), intervenções (Santos \& Fabbro, 2018) e segurança (Santos \& Fabbro, 2018) (Figura 4).

Figura 4 - Palavras-chave que retratam resumidamente fatores que influenciam a não realização do parto via vaginal com base na assistência profissional, no Sistema Único de Saúde (SUS) no Brasil; publicações de 2016 a 2021.

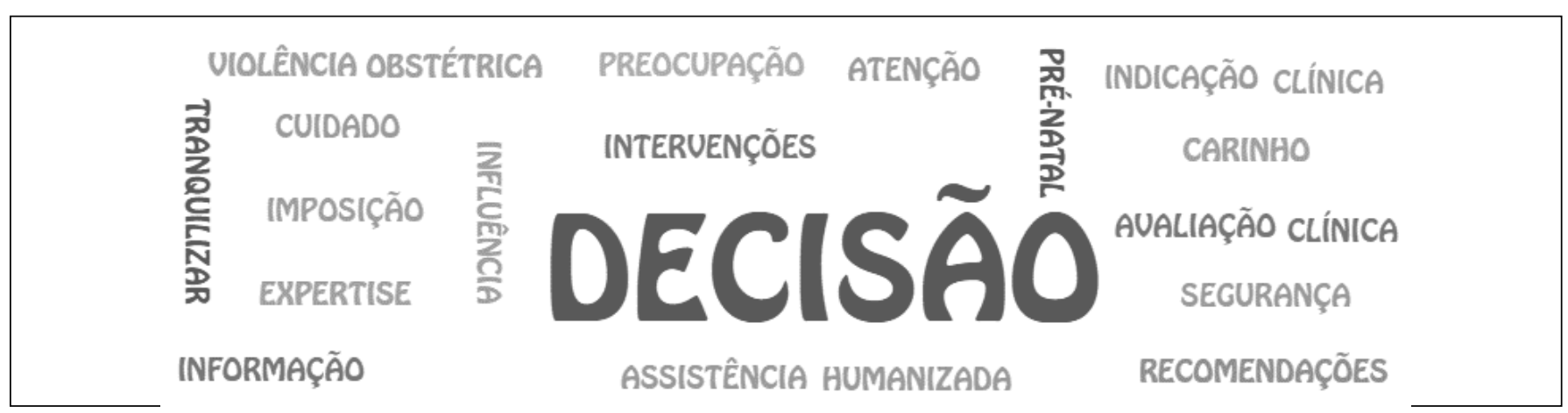

Fonte: Autores.

\section{Discussão}

\section{Fatores para a não realização do parto via vaginal com base no comportamento materno}

O estudo de Albuquerque et al. (2019) nos traz como resultado que enfermeiras observam que a insegurança é uma representação social significante entre gestantes, em diferentes contextos; uma das formas de expressão é a partir do medo do parto normal. O medo também é citado por Silva et al. (2019) em outro artigo analisado como um dos principais motivos para a mulher preferir o parto cesárea. No próprio ponto de vista das gestantes, em acompanhamento pré-natal, no imaginário de como será a experiência de um possível parto normal, além do sentido da dor como pressuposto de sofrimento, também declaram sentimentos de inquietação expressos por medo e insegurança (Almeida et al.,2012). 
Um achado que remete aos sentimentos indesejados, evidencia que durante o trabalho de parto as mulheres experimentam sentimentos negativos, como desconforto, abandono, negligência e insatisfação - esta gerada por ausência de acompanhamento contínuo, verticalização das relações, ausência de acompanhamento profissional e banalização da dor (Bomfim et al., 2021). Diante desses sentimentos, em um estudo realizado numa maternidade pública de Teresina-Piauí, Cavalcanti Cavalcante, Oliveira, Ribeiro e Nery (2007) declaram que para as mulheres, a experiência do parto foi traumática, onde expressaram medo, tristeza, ansiedade e carência de atendimento humanizado.

Quando relacionado ao parto cesáreo, um dos artigos aponta que as gestantes têm confiança no profissional, desencadeando conformismo perante a informação dada, mesmo que seja superficial; sobretudo, concordância com a decisão do profissional de realizar a conduta cirúrgica (Barral et al., 2020). Neste contexto, podemos verificar em outro estudo, que por mais que a vontade seja o parto via vaginal, as mulheres contribuem passivamente para a reprodução do modelo biomédico e intervencionista, principalmente quando aceitam a conduta imposta sem a intensão de mudar (Freire et al., 2011). Em um estudo de delineamento transversal, em um hospital de atendimento público e privado, selecionado sob a proposta de pesquisa, destaca que o desejo por cesariana no início da gestação ou não ter preferência de parto definida, fatores obstétricos e o parto em detrimento a fatores sociodemográficos, tem associação com maior realização de cesariana; sobretudo quanto maior a escolaridade, maior a prevalência de cesariana (Rasador et al., 2019).

Neste sentido, Carniel et al., (2007), também encontraram associação entre o parto cesáreo, nas condições de gestação dupla, primíparas e multíparas; onde as chances para indicação de cesariana foram maiores para mulheres com melhor nível socioeconômico e com pré-natal adequado, todavia, a indicação não se baseou somente em normas técnicas, mas também em razões não-médicas. Dentre os achados de Rasador et al., (2019), um dos estudos analisados, a prevalência de cesárea foi quase seis vezes maior em mulheres com cesárea prévia, sendo para o estudo o principal fator de risco para nova cesárea. Todavia, uma explicação possível para a realização da cesárea é o receio da ocorrência de ruptura uterina.

Diante desta condição discute-se com o estudo de Câmara et al. (2016), os quais trazem que a história de cesariana não se configura indicação para parto cesárea; no entanto, nestes casos há um pequeno aumento no risco de rotura uterina durante o trabalho de parto, levando em consideração outros fatores, como por exemplo, a quantidade de cesáreas prévias, o tipo de histerotomia e o intervalo entre a última cesariana e o parto normal. De acordo com um resultado encontrado, a mulher ainda está inserida em uma pseudoautonomia, que ocorre devido a uma lacuna existente no processo de orientação e preparo da mulher para sua decisão, e por consequência a impede de assumir o protagonismo na escolha do parto desejado, que por muitas vezes acaba por se submeter a uma escolha que nem sempre é necessária e/ou benéfica (Silva et al., 2019). Em consonância com o achado, a decisão da mulher na escolha da via de parto está se tornando uma questão de opção (e não uma indicação técnica) sem conhecimento e orientação, embasada em uma indicação e consequentemente a diminuição da autonomia da mulher em detrimento a via do seu parto (Weidle et al., 2014).

Alguns elementos dificultadores para a escolha do parto normal embasado no comportamento materno mencionados no estudo de Santos et al., (2018) se delineiam pela idealização do parto perfeito, imaginário social do parto, vulnerabilidades e fragilidades da mulher no parto e cultura da cesárea. Partindo do princípio deste último elemento, o estudo de Copelli et al., (2015) se refere a confirmação da cultura de cesárea existente no Brasil, onde demonstram que, apesar das gestantes preferirem o parto vaginal, independente do seu perfil social, durante o pré-natal ou no trabalho de parto grande parte das mulheres mudam de posicionamento e optam por realizar o parto cesárea.

Destacando o ponto de vista de outros autores sobre a idealização do parto perfeito (Santos \& Fabbro, 2018), geralmente a mulher idealiza como será o seu parto, quando acontece de forma diferente surge a decepção. Com o ocorrido inesperado, há mulheres que apresentam frustração e até mesmo sentimento de fracasso, entendendo que falhou ou fez tudo errado. Este tipo de evento acontece quando a mulher forma para si um imaginário imutável sobre o parto, sem levar em conta 
que o imprevisível e a possibilidade de que possa ocorrer de forma inteiramente diversa (Consonni et al., 2003). Contextualizando um (imaginário social do parto) dos elementos dificultadores para a escolha do parto normal, mencionado por Santos e Fabbro (2018), Campos et al. (2020) dizem que uma das opções para a gradativa mudança do imaginário social/cultural do parto é a sensibilização dos profissionais que assistem ao parto, diante do sentimento subjetivo da dor da parturiente, empregando assim, um repasse de experiência positiva à mulher durante o processo.

Em outra vertente, com os achados de vulnerabilidades e fragilidade encontrados no estudo de Santos e Fabbro (2018), é possível observar o impacto que estes elementos dificultadores desencadeiam na condição parturitiva da mulher. Nesse sentido, Cabral, Hirt e Sand (2013) destacam que as altas taxas de parto cesárea e morbimortalidade materna e perinatal são representativos de algumas situações de vulnerabilidades de que as mulheres estão inseridas tanto no período gravídico, quanto puerperal. Por outro lado, Souza e Gualda (2016) ressaltam que a fragilidade também se faz presente no período gestacional e de parto da mulher, tendo em vista que neste momento, o apoio dos profissionais se faz importantes, tanto quando relacionado a mulher quanto do seu acompanhante.

\section{Fatores para a não realização do parto via vaginal com base na assistência profissional}

Com base no estudo de Albuquerque et al (2019) vivenciar o parto via vaginal é desejado, pautado por ser um evento natural, permeado pelo auxílio divino no processo de evolução. Sobretudo, também é ressaltado que este evento é ancorado por insegurança e imposição dos serviços públicos, o que acaba por impossibilitar que este desejo aconteça. Nesta perspectiva de que grande parte das mulheres de fato não têm escolha e não decidem sobre a via de parto, é visto mais uma vez que a decisão cabe aos profissionais de saúde. Mais especificamente no âmbito do Sistema Único de Saúde mulheres que experienciam a cirurgia cesárea relatam ser uma indicação médica (Velho et al., 2014).

No estudo de Barral et al. (2020), os discursos das entrevistadas evidenciam que a decisão pela via de parto foi exclusivamente do profissional médico, sem a participação da gestante, sendo a avaliação clínica o elemento-chave para a conduta cirúrgica, todavia, as justificativas não eram necessariamente indicações absolutas de cesárea. Neste contexto, com a contribuição de Silva, Prates e Campelo (2014), fica clara a importância do papel da mulher como portadora da decisão a respeito da via de parto de sua preferência; sobretudo, cabe aos profissionais de saúde, principalmente os que realizarão o parto, aceitar ou interferir na escolha da mãe, somente quando a conduta trouxer risco a mãe e/ou ao feto.

Para o processo de parto via cesárea, de acordo com resultados encontrados, o cuidado - preocupação, carinho e atenção, prestado por uma assistência humanizada, transmite segurança e confiança para a gestante, sobretudo a assistência profissional pode contribuir para tranquilizar a paciente e fazer com que se sinta bem e protegida (Barral et al., 2020). Perante esta colocação, Velho et al. (2014) contribui reafirmando que a avaliação positiva no atendimento prestado, realizado de forma atenciosa e calma, com cuidado e orientação, em atitudes carinhosas, que auxiliam e promovem apoio e conforto, desencadeiam a tranquilidade, segurança e atendimento, sobretudo, situação a qual que subsidia tanto quando relacionado o parto normal quanto o parto cesáreo.

O artigo de Rasador e Abegg (2019), incluído na pesquisa, mostra que uma das variáveis fortemente associada ao aumento da prevalência de cesariana, relaciona-se ao pré-natal. Neste sentido, Oliveira et al., (2016) salientam que é necessário investir na formação dos profissionais de saúde, capacitando-os para realizar o acompanhamento e o cuidado pré-natal, além de visualizar apenas o parto normal, e intervir com a cirurgia cesárea em casos que realmente são necessários, garantindo a humanização do atendimento, as boas práticas e a promoção do parto e nascimento seguros. Um dos artigos analisados, demonstra que a principal fonte de informações sobre a gravidez e parto é o médico, o qual informa que tanto o parto normal quanto a cesárea são seguros. No entanto, o médico é pautado como o principal fator influência para a realização do parto cesárea. Já as gestantes que manifestam preferência por esta via de parto, dois dos fatores que contribuem para essa escolha são 
em decorrência à indicação clínica e recomendações médicas, o que acaba por entender que a decisão do profissional de saúde tem um peso significativo na decisão da gestante sobre qual a via de parto (Silva et al., 2019).

Neste sentido, embasando a posição de outros autores, faz-se uma ressalva sobre possibilidades, nas quais o profissional enfermeiro obstetra pode implementar função contrária, prestando papel essencial para apoiar a mulher na evolução do trabalho de parto, ampliando seus conhecimentos sobre o momento, para assim consequentemente reduzir as influências externas que direcionam para um parto com intervenções. A atuação destes profissionais no planejamento e implementação de práticas educativas interdisciplinares, em especial em grupos de gestantes, também é de suma importância para este processo (Copelli et al., 2015). Um elemento dificultador para a escolha do parto normal alicerçado na assistência profissional citado no artigo de Santos e Fabbro (2018) é a violência obstétrica/intervenções desnecessárias. Paralelamente a esta condição Copelli et al. (2015) contextualizam afirmando que além da desinformação, as intervenções desnecessárias durante o trabalho de parto e as violências obstétricas, transformam o que era para ser uma ação natural em um procedimento desumanizado, contribuindo ainda mais para as sensações dolorosas e medos, acarretando consequentemente a aceitação e até mesmo solicitação da cirurgia cesárea.

Os resultados encontrados nos estudos de Barral et al. (2020) e Santos et al., (2018) destacam a relação entre a gestante, a informação/conhecimento e o profissional. Corroborando com isso, Barral et al. (2020) mencionam que a expertise do profissional médico somada à falta de conhecimento da gestante faz com que a mulher confie no que está sendo dito e não questione a conduta da cesárea e que essa desinformação, muitas vezes coloca a mulher em situação de vulnerabilidade. Por outro lado, Santos e Fabbro (2018) relatam que se a mulher busca e tem acesso à informação e está ampara por profissionais que lhe transmita segurança, é possível obter escolhas mais conscientes sobre o parto. Nestas perspectivas, Sodré, Merighi e Bonadio (2012) contribuem dizendo que estimular a tomada de decisão informada da mulher dispõe de critérios ímpios na vida dessas pessoas, respeitando o direito do próprio corpo, valorização da capacidade de decidir, autonomia e promoção do direito à pessoa.

Com a seleção e análise das pesquisas, constatou-se que é existente a falta de pesquisas na região Centro-Oeste e Norte do Brasil, centralizando a maior parte dos estudos na região Nordeste, a qual segundo o IPEA ([Instituto de Pesquisa Econômica Aplicada], 2015) é a região mais pobre do país; todavia, a região mais rica - o Sudeste (IPEA, 2015), apresenta uma posição abaixo na quantidade de pesquisas realizadas nesta linha de conteúdo, sendo a região Sul em última colocação, é possível que o número de publicações esteja relacionado ao número de universidades e de periódicos disponíveis.

\section{Conclusão}

Com base no comportamento materno o fator medo é o principal contribuinte para a não realização do parto via vaginal. Este sentimento é seguido equiparadamente, pela insegurança, desconforto, abandono, negligência, insatisfação, confiança, conformismo, concordância, desejo, obstétrico, sociodemográfico, escolaridade, cesárea prévia, ruptura uterina, pseudoautonomia, idealização, imaginário, vulnerabilidade, fragilidade, cultura, informação, conhecimento e desinformação.

Em contrapartida, quando relacionado a assistência profissional, ressalta-se que o fator decisão foi o mais mencionado como contribuinte para a não realização do parto via vaginal. Os seguintes fatores também são apresentados como contribuição (na mesma proporção): imposição, avaliação clínica, cuidado, preocupação, carinho, atenção, assistência humanizada, tranquilizar, pré-natal, informação, influência, indicação clínica, recomendações, violência obstétrica, intervenções, expertise e segurança.

É válido ressaltar que cada fator mencionado tanto com base no comportamento materno quanto na assistência profissional, se compromete à um contexto de realidade, podendo estar relacionado a experiências/assistências em tempos de 
pré-natal, parto e/ou puerpério, no contexto da vivência do parto via vaginal e/ou cirurgia cesárea. Com a metodologia utilizada e análise dos estudos resultantes, observa-se que não houveram pesquisas realizadas na região Centro-oeste e Norte, nesta linha de conteúdo.

Os resultados obtidos nos proporcionam identificarmos as condições que possibilitam ou dificultam a realização do parto via vaginal, com isso, o estudo contribui eficazmente para a assistência da enfermagem na atenção à gestante, parturiente e puérpera. A perspectiva foi detectar quais as competências e fragilidades que estão acorrendo na prestação da assistência dos profissionais do Sistema Único de Saúde, o que consequentemente contribuirá para a elaboração de planejamento e implementação melhorada do serviço da enfermagem a este público.

Sobretudo, é visto que muitas práticas se revertidas podem fazer diferença devastadora na realidade desta epidemia de cesárea que ocorre atualmente no Brasil. Sugere-se a realização de pesquisas nas regiões Centro-oeste e Norte, onde os estudos ainda são escassos, para uma possível visualização do cenário da região e posteriormente a obtenção de constante melhora do quadro; a opção de novos estudos identificando quais os reais motivos registrados em documentos que subsidiam a realização do parto cesáreo, para uma nova avaliação do que está acontecendo e elencar estratégias para que a realidade de exorbitante realização de cesárea, mude.

\section{Referências}

Albuquerque, N. L. A., Mendonça, E. F., Guerra, M. C. G. C., Silva, J. C. B., \& Lins, H. N. S. (2019). Representações Sociais de Enfermeiras da Atenção Básica sobre o Parto Normal. Revista Ciência Plural, 5(1):34-51. https://pesquisa.bvsalud.org/portal/resource/pt/biblio-1007338

Almeida, N. A. M., Medeiros, M., \& Souza, M. R. (2012). Perspectivas de Dor do Parto Normal de Primigestas no Período Pré-Natal. Texto Contexto Enferm. https://www.scielo.br/j/tce/a/nNjD3NdDkgLjkV9QzCfySmw/?format=pdf\&lang=pt

Anjos, C. S., Westphal, F., \& Goldman, R. E. (2014). Cesárea Desnecessária no Brasil: Revisão integrativa. ABENFO Enfermagem Obstétrica, p. 86-94. 2014. http://www.enfo.com.br/ojs/index.php/EnfObst/article/download/21/19

Barral, F. E., Couto, T. M., Almeida, L. C. G., Bispo, T. C. F., Oliveira, G. M., \& Webler, N. (2020). Parto Cirúrgico: As Múltiplas Experiências de Mulheres. Rev baiana enferm. 34:e38128. http://www.revenf.bvs.br/pdf/rbaen/v34/1984-0446-rbaen-34-e38128.pdf

Betran, A. P., Torloni, M. R., Zhang, J., Ye, J., Mikolajczyk, R., Deneux-Tharaux, C., Oladapo, O. T., Souza, J. P., Tunçalp, Ö., Vogel, J. P., \& Gülmezoglu, A. M. (2015). Qual é a taxa ideal de cesariana em nível populacional? Uma revisão sistemática de estudos ecológicos. Biblioteca Nacional de Medicina dos EUA. BioMed Central. https://www.ncbi.nlm.nih.gov/pmc/articles/PMC4496821/

Bomfim, A. N. A., Couto, T. M., Lima, K. T. R. S., Almeida, L. T. S., Santos, G. O., \& Santana, A. T. (2021). Percepções de Mulheres sobre a Assistência de Enfermagem Durante o Parto Normal. Rev baiana enferm, 35:e39087. http://www.revenf.bvs.br/scielo.php?script=sci_arttext\&pid=S217886502021000100316

Brasil. Ministério da Saúde. (2017). Secretaria de Ciência, Tecnologia e Insumos Estratégicos. Departamento de Gestão e Incorporação de Tecnologias em Saúde. Diretrizes nacionais de assistência ao parto normal: versão resumida [recurso eletrônico] / Ministério da Saúde, Secretaria de Ciência, Tecnologia e Insumos Estratégicos, Departamento de Gestão e Incorporação de Tecnologias em https://bvsms.saude.gov.br/bvs/publicacoes/diretrizes_nacionais_assistencia_parto_normal.pdf

Brasil. Ministério da Saúde. (2015). Diretrizes de Atenção à Gestante: a operação Cesariana. Secretaria de Ciência, Tecnologia e Insumos Estratégicos. CONITEC (Comissão Nacional de no nerporação de http://conitec.gov.br/images/Consultas/Relatorios/2015/Relatorio_PCDTCesariana_CP.pdf

Brasil. Ministério da Saúde. (2016). Secretaria de Atenção à Saúde - Portaria no 306 de 28 de março de 2016. Aprova as Diretrizes de Atenção à Gestante: a operação cesariana. Diário oficial da União. ed. 59, s. 1, p 58. http://www.in.gov.br/materia/-/asset_publisher/Kujrw0TZC2Mb/content/id/22554355

Brenes, A. C. (1991). História da Parturição no Brasil, Século XIX. Cadernos de Saúde Pública, 7 (2): 135 - 149. http://www.scielo.br/pdf/csp/v7n2/v7n2a02.pdf

Cabral, F. B., Hirt, L. M., \& Sand, I. C. P. V. (2013). Atendimento Pré-natal na Ótica de Puérperas: da Medicalização à Fragmentação do Cuidado. Rev Esc Enferm. https://www.scielo.br/j/reeusp/a/pcWQx4DnHfTpnzQTY7JXXXB/?lang=pt\&format=pdf

Câmara, R., Burlá, M., Ferrari, J., Lima, L., Junior, J. A., Braga, A., \& Filho, J. R. (2016). Cesariana a Pedido Materno. Rev. Col. Bras. Cir. https://www.scielo.br/j/rcbc/a/44yLTZ766jrmDJLCD3XxqrM/?lang=pt\&format=pdf

Campos, V. S., Morais, A. C., Araújo, P. O., Morais, A. C., Almeida, B. S., \& Silva, J. S. (2020). Experiência de Puérperas com a Dor do Parto Normal. Revista Eletrônica Acervo Saúde / Electronic Journal Collection Health. Recuperado de https://doi.org/10.25248/reas.e2396.2020 
Research, Society and Development, v. 11, n. 3, e49611326810, 2022

(CC BY 4.0) | ISSN 2525-3409 | DOI: http://dx.doi.org/10.33448/rsd-v11i3.26810

Carniel, E. F., Zanolli, M. L., \& Morcillo, A. M. (2007). Fatores de Risco para Indicação do Parto Cesáreo em Campinas (SP). Rev Bras Ginecol Obstet. https://www.scielo.br/j/rbgo/a/p593cSCQZ9xHbcJgKMSPszq/?lang=pt\&format=pdf

Cavalcante, F. N., Oliveira, L. V., Ribeiro, M. M. O. M., \& Nery, I. S. (2007). Sentimentos vivenciados por mulheres durante trabalho de parto e parto. Salvador: Revista Baiana de Enfermagem, 21(1), 31-40. https://doi.org/10.18471/rbe.v21i1.3910

Consonni, E. B., Calderon, I. M. P., Consonni, M., \& Rudge, M. V. C. (2003). Aspectos Psicológicos na Gravidez e Parto. Femina. https://www.researchgate.net/profile/Elenice-

Consonni/publication/236893279_Aspectos_psicologicos_na_gravidez_e_parto/links/56db621f08aebe4638beef57/Aspectos-psicologicos-na-gravidez-eparto.pdf

Copelli, F. H. S., Rocha, L., Zampieri, M. F. M., Gregório, V. R. P., \& Custódio, Z. A. O. (2015). Fatores Determinantes para a Preferência da Mulher pela Cesariana. Texto Contexto Enferm. https://www.scielo.br/j/tce/a/rF5JT3cxSzyrQbZjL76mgVP/?lang=pt\&format=pdf

Freire, N. C., Nunes, I. M., Almeida, M. S., \& Gramacho, R. C. C. V. (2011). Parto Normal ou Cesárea? A Decisão na Voz das Mulheres. Revista Baiana de Enfermagem. https://periodicos.ufba.br/index.php/enfermagem/article/download/6027/4900

HUMAP - Hospital Universitário Maria Aparecida Pedrossian. (2018). Protocolos de Obstetrícia HUMAP: Protocolo de Antecipação Eletiva do Parto e Indicação de Cesariana. UFMS. http://www2.ebserh.gov.br/documents/17082/3273530/Antecipa\%C3\%A7\%C3\%A3o+Eletiva+do+Parto+e+Indica\%C3\%A 7\%C3\%A3o+de+Cesariana.pdf/35d8bec5-96e0-42f9-b88c-18783d15a9b5

IPEA - Instituto de Pesquisa Econômica Aplicada. (2015). Fatos Recentes do Desenvolvimento Regional no Brasil. Ipea. https://www.gov.br/secretariadegoverno/pt-br/portalfederativo/biblioteca-federativa/estudos/td_2054-desenvolvimento-regional.pdf

Maldonado, M. T. (2002). Psicologia da Gravidez: parto e puerpério. (16a ed.), Saraiva.

Mendes, K. D. S., Silveira, R. C. C. P., \& Galvão, C. M. (2008). Revisão Integrativa: Método de Pesquisa para a Incorporação de Evidências na Saúde e na Enfermagem. Texto Contexto Enferm. https://www.scielo.br/pdf/tce/v17n4/18.pdf

Oliveira, R. R., Melo, E. C., Novaes, E. S., Ferracioli, P. L. R. V., \& Mathias, T. A. F. (2016). Fatores associados ao parto cesárea nos sistemas público e privado de atenção à saúde. Rev Esc Enferm USP, 50(5):734-741. http://www.scielo.br/pdf/reeusp/v50n5/pt_0080-6234-reeusp-50-05-0734.pdf

OMS - Organização Mundial da Saúde. (2015). Declaração da OMS sobre Taxas de Cesáreas. WHO/RHR/15.02. https://apps.who.int/iris/bitstream/handle/10665/161442/WHO_RHR_15.02_por.pdf?sequence=3

Pimentel, T. A., \& Oliveira-Filho, E. C. (2016). Fatores que influenciam na escolha da via de parto cirúrgica: uma revisão bibliográfica. Universitas: Ciências da Saúde, v. 14, n. 2, p. 187-199. https://www.publicacoesacademicas.uniceub.br/cienciasaude/article/download/4186/3279

Rasador, S., \& Abegg, C. (2019). Fatores associados à via de parto em um município da região nordeste do Estado do Rio Grande do Sul, Brasil. Rev. Bras. Saúde Mater. Infant, 19 (4): 807-815. https://www.scielo.br/pdf/rbsmi/v19n4/pt_1519-3829-rbsmi-19-04-0797.pdf

Rissin, A., \& Filho, M. B. (2018). A OMS e a epidemia de cesarianas. Rev. Bras. Saúde Mater. Infant. http://www.scielo.br/pdf/rbsmi/v18n1/pt_1519-3829rbsmi-18-01-0003.pdf

Santos, S., \& Fabbro, M. R. C. (2018). A Difícil Tarefa de Escolher o Parto Natural. Ciencia Y Enfermeria. https://scielo.conicyt.cl/pdf/cienf/v24/0717-9553cienf-24-11.pdf

Silva, M. M. J., Silva, S. C. B., \& Melo, G. A. (2019). Autonomia da gestante na escolha do tipo de parto. Investigación en Enfermería: Imagen y Desarrollo, https://revistas.javeriana.edu.co/index.php/imagenydesarrollo/article/view/19754

Silva, S. P. C., Prates, R. C. G. \& Campelo, B. Q. A. (2014). Parto Normal ou Cesariana? Fatores que Influenciam na Escolha da Gestante. Rev Enferm UFSM. https://periodicos.ufsm.br/reufsm/article/download/8861/pdf

Sodré, T. M., Merighi, M. A. B., \& Bonadio, I. C. (2012). Escolha Informada no Parto: Um Pensar para o Cuidado Centrado nas Necessidades da Mulher. Cienc Cuid Saude. https://periodicos.uem.br/ojs/index.php/CiencCuidSaude/article/view/17062/pdf

Souza, S. R. R. K., \& Gualda, D. M. R. (2016). A Experiência da Mulher e de seu Acompanhante no Parto em uma Maternidade Pública. Texto Contexto Enferm. https://www.scielo.br/j/tce/a/Sg7K3tTsB4MHLWZm4mH4tTs/?lang=pt\&format=pdf

Velho, M. B., Santos, E. K. A., \& Collaço, V. S. (2014). Parto normal e cesárea: representações sociais de mulheres que os vivenciaram. Rev Bras Enferm, 67(2): 282-9. http://www.scielo.br/pdf/reben/v67n2/0034-7167-reben-67-02-0282.pdf

Vendrúscolo, C. T., \& Kruel, C. S. (2015). A História do Parto: do Domicílio ao Hospital; das Parteiras ao Médico; de Sujeito a Objeto. Disciplinarum Scientia, 16(1), 95-107. file:///C:/Users/karla/Downloads/1842-5134-2-PB.pdf

Viana, T. G. F., Martins, E. F., Sousa, A. M. M., Souza, K. V., Rezende, E. M., \& Matozinhos, F. P. (2018). Motivo da Realização de Cesárea Segundo Relato das Mães e Registros de Prontuários em Maternidades de Belo Horizonte. Rev Min Enferm, 22:e-1073. http://www.dx.doi.org/10.5935/1415-2762.20180003

Weidle, W. G., Medeiros, C. R. G., Grave, M. T. Q., \& Bosco, S. M. D. (2014). Escolha da Via de Parto pela Mulher: Autonomia ou Indução? Cad. Saúde Colet. Recuperado de https://www.scielo.br/j/cadsc/a/wRvpVrnwmPcqVLqJTLLcvbb/?format=pdf\&lang=pt 